\title{
Correction to: Approximate recovery of residual displacement from the strong motion recordings of the 24 August 2016 Amatrice, Italy earthquake
}

\section{Reeves Whitney ${ }^{1}$}

Published online: 1 December 2017

(C) Springer Science+Business Media B.V., part of Springer Nature 2017

\section{Correction to: Bull Earthquake Eng}

https://doi.org/10.1007/s10518-017-0273-9

Unfortunately an error was introduced during typesetting. In Eq. (6) $\Sigma$ should be $\ni$. The correct Eq. (6) should read:

$$
t_{1} \ni \min \left\{\int_{0}^{t_{d}}\left[v_{a d j}(t)\right]^{2} d t\right\}
$$

The original article can be found online at https://doi.org/10.1007/s10518-017-0273-9.

\section{Reeves Whitney}

reeves.whitney@manhattan.edu

1 Department of Civil and Environmental Engineering, Manhattan College, 4513 Manhattan College Parkway, Riverdale, NY 10471, USA 